\title{
The impact of adjuvant therapy on contralateral breast cancer risk and the prognostic significance of contralateral breast cancer: a population based study in the Netherlands
}

\author{
Michael Schaapveld • Otto Visser - W. J. Louwman - Pax H. B. Willemse • \\ Elisabeth G. E. de Vries · Winette T. A. van der Graaf · Renée Otter • \\ Jan Willem W. Coebergh · Flora E. van Leeuwen
}

Received: 19 July 2007 / Accepted: 20 July 2007/Published online: 9 August 2007

(C) Springer Science+Business Media, LLC 2007

\begin{abstract}
Background The impact of age and adjuvant therapy on contralateral breast cancer (CBC) risk and prognostic significance of $\mathrm{CBC}$ were evaluated. Patients and Methods In 45,229 surgically treated stage I-IIIA patients diagnosed in the Netherlands between 1989 and 2002 CBC risk was quantified using standardised incidence ratios (SIRs), cumulative incidence and Cox regression analysis, adjusted for competing risks. Results Median follow-up was 5.8 years, in which 624 CBC occurred
\end{abstract}

M. Schaapveld $(\bowtie) \cdot$ R. Otter

Comprehensive Cancer Center North-Netherlands (CCCN), P.O. Box 330, Groningen 9700 AH, The Netherlands

e-mail: m.schaapveld@ikn.nl

O. Visser

Comprehensive Cancer Center Amsterdam (CCCA),

P.O. Box 9236, Amsterdam 1006 AE, The Netherlands

W. J. Louwman · J. W. W. Coebergh

Comprehensive Cancer Center South (CCCS), P.O. Box

231, Eindhoven 5600 AE, The Netherlands

P. H. B. Willemse · E. G. E. de Vries

Department of Medical Oncology, University of Groningen and University Medical Center Groningen, P.O. Box 30.001,

Groningen $9700 \mathrm{RB}$, The Netherlands

W. T. A. van der Graaf

Department of Medical Oncology, Radboud University

Nijmegen Medical Centre, P.O. Box 9101, Nijmegen 6500 HB,

The Netherlands

J. W. W. Coebergh

Department of Public Health, Erasmus University Medical Center, P.O. Box 2040, Rotterdam 3000 CA, The Netherlands

F. E. van Leeuwen

Department of Epidemiology, Netherlands Cancer Institute,

Plesmanlaan 121, Amsterdam 1066 CX, The Netherlands
$<6$ months after the index cancer (synchronous) and 1,477 thereafter (metachronous). Older age and lobular histology were associated with increased synchronous $\mathrm{CBC}$ risk. Standardised incidence ratio (SIR) of CBC was $2.5(95 \%$ confidence interval (95\% CI) 2.4-2.7). The SIR of metachronous CBC decreased with index cancer age, from 11.4 (95\% CI 8.6-14.8) when <35 to 1.5 (95\% CI 1.4-1.7) for $\geq 60$ years. The absolute excess risk of metachronous CBC was 26.8/10,000 person-years. The cumulative incidence increased with $0.4 \%$ per year, reaching $5.9 \%$ after 15 years. Adjuvant hormonal (Hazard rate ratio (HR) 0.58; 95\% CI 0.48-0.69) and chemotherapy (HR 0.73; 95\% CI 0.60-0.90) were associated with a markedly decreased CBC risk. A metachronous $\mathrm{CBC}$ worsened survival (HR 1.44; 95\% CI 1.33-1.56). Conclusion Young breast cancer patients experience high synchronous and metachronous $\mathrm{CBC}$ risk. Adjuvant hormonal or chemotherapy considerably reduced the risk of $\mathrm{CBC}$. $\mathrm{CBC}$ occurrence adversely affects prognosis, emphasizing the necessity of long-term surveillance directed at early CBC-detection.

Keywords Adjuvant systemic therapy .

Contralateral breast cancer · Prognosis - Radiotherapy

\section{Introduction}

Breast cancer is the most common malignancy in women in North America and Western Europe. Contralateral breast cancer (CBC) is the most frequent second cancer reported after primary breast cancer, comprising between 30 and $50 \%$ of all second cancers [1-5]. Breast cancer patients appear to have a two- to threefold increased risk of developing $\mathrm{CBC}$. Previous studies observed a constant increase in $\mathrm{CBC}$ risk over time, up to a cumulative risk of 
5-7\% 10 years after diagnosis [6-9]. However, most studies included patients diagnosed and treated more than 15-20 years ago while indications for adjuvant treatment have changed since.

Factors such as family history, young age and lobular histology have been associated with increased CBC risk [6, $8,10-12]$. There is increasing evidence that adjuvant treatment affects CBC risk. A meta-analysis by the Early Breast Cancer Trialists' Collaborative Group (EBCTCG) showed that tamoxifen for 2 or 5 years resulted in proportional reductions of the CBC incidence of $26 \%$ and $47 \%$, respectively [13]. Recent data suggest that aromatase inhibitors may be even more effective in reducing CBC [14-16]. The EBCTCG meta-analyses showed also a marginally significant $20 \%$ decrease in CBC risk after adjuvant chemotherapy [17]. Radiation doses to the contralateral breast are generally fairly low and rather unlikely to confer an increased CBC risk. However, a large casecontrol study did find an increased radiation-associated risk among long-term breast cancer survivors diagnosed between 1935 and 1982, who received radiotherapy before age 45 [18]. A recent analysis of SEER data also found a slightly increased CBC risk after radiotherapy in patients aged $<45$ years, surviving longer than 5 years [6].

No recent data are available investigating the effects of current breast cancer therapy on CBC risk among unselected breast cancer patients. The effects of current adjuvant treatment options on $\mathrm{CBC}$ occurrence and the impact of $\mathrm{CBC}$ on patient survival are important and can provide guidance for follow-up. Therefore we analysed CBC occurrence in a large population-based cohort of Dutch breast cancer patients, diagnosed between 1989 and 2002, evaluating the impact of age and treatment of the index cancer and time since diagnosis on $\mathrm{CBC}$ risk. In addition the prognostic significance of $\mathrm{CBC}$ was studied.

\section{Methods}

\section{Patients}

The cohort comprises all surgically treated stage I-IIIA (comprising TNM stages up to and including T3N1M0) primary breast cancer patients diagnosed in the Comprehensive Cancer Centre North (CCCN), Amsterdam (CCCA) and South (CCCS) regions from January 1989 until January 2003. Their cancer registries cover the northwestern and southeastern part of the Netherlands, an area with 7.2 million inhabitants (45\% of the Dutch population). The cohort was composed in accordance to privacy regulations of the Netherlands Cancer Registry (NCR).
Data collection

PALGA, the nationwide Dutch network and registry of histo- and cytopathology submits reports of diagnosed malignancies to the Dutch cancer registries. The national hospital databank, which receives discharge diagnoses of all Dutch hospitals, completes case ascertainment. Registry personnel collect data on diagnosis, stage and treatment from the medical records using the registration and coding manual of the NCR. All Dutch patients are treated in public hospitals. Vital status and date of death were established from the patient's medical record or through linkage with municipal population registries or the national death registry of the Central Bureau for Genealogy (CBG). Date of censoring was set at 12-31-2004.

Stage was based on pathological information or clinical information if pathology data were missing $[19,20]$. All primary cancers before or after the index breast cancer were ascertained through a computerised record linkage method. Patients with invasive breast cancer following a non-invasive breast cancer, treated with ablation or amputation, and patients with a previous invasive cancer other than non-melanoma skin cancer were excluded. Any $\mathrm{CBC}$, not classified as a recurrence, was considered a primary CBC. Patients who presented with $\mathrm{CBC}$ and distant metastases at diagnosis of this $\mathrm{CBC}$ were included. Information on the occurrence of metastases during follow-up was incomplete in the registries and determining whether these metastases were due to the index cancer or due to the CBC was not otherwise possible. To facilitate comparison with international literature non-invasive $\mathrm{CBC}$ was excluded as event. Non-invasive CBC comprised about $10 \%$ of all $\mathrm{CBC}$ and some $5 \%$ of the non-invasive $\mathrm{CBC}$ was followed by an invasive CBC. Patients who developed a noninvasive $\mathrm{CBC}$, treated with ablation or amputation, were censored at date of diagnosis of this tumour. An invasive $\mathrm{CBC}$ diagnosed within 6 months of the index cancer was defined as synchronous, all others as metachronous CBC.

\section{Treatment}

The prevailing treatment guidelines during the study period are outlined below. Breast conserving surgery with axillary lymph node dissection was indicated for tumours $<4 \mathrm{~cm}$, complemented with radiotherapy to the breast. Alternatively, a modified radical mastectomy was performed. Loco-regional radiotherapy, consisting of parasternal, axillary, infra and supra-clavicular nodal irradiation, was indicated in case of $>3$ positive axillary nodes or extranodal growth. Irradiation of the parasternal nodes was indicated for node-positive patients with a medially located tumour. Pre-menopausal node-positive patients received 
adjuvant chemotherapy, generally consisting of cyclophosphamide, methotrexate and 5-fluorouracil. Anthracyclinebased chemotherapy was increasingly used for high-risk patients since the mid-1990s. Until 1998 post-menopausal node-positive patients received 2 years tamoxifen. Since 1998, adjuvant systemic therapy was also administered to node-negative patients with less favourable tumour characteristics (intermediate or poorly differentiated tumours $\geq 2 \mathrm{~cm}$ ). Furthermore, since 1998 all hormone-receptorpositive node-positive and unfavourable node-negative patients were to receive 5-years tamoxifen, irrespective of menopausal status. Inoperable and locally advanced cancers received chemotherapy, hormonal therapy and/or radiotherapy.

\section{Statistical analysis}

$\mathrm{CBC}$ risk was quantified using various risk measures. The standardised incidence ratio (SIR) compares the observed with expected numbers of $\mathrm{CBC}$ based on age- and calendar-year specific breast cancer incidence rates for the Dutch female population. The 1989, 1990, 1992, 1994, 1996, 1998, 2000 and 2002 incidence rates were derived from the NCR and used to compute expected CBC numbers. Time at risk started at the date of index cancer diagnosis for synchronous $\mathrm{CBC}$ and 6 months thereafter for metachronous $\mathrm{CBC}$ and ended at the date of $\mathrm{CBC}$ diagnosis, the date of death or the date of last linkage with the municipal population registry or $\mathrm{CBG}$. No information on prophylactic contralateral mastectomies was available. The CIs for the SIRs were calculated assuming a Poisson distribution for the observed number of CBC. SIRs were computed by age at diagnosis $(<35,35-39,40-49,50-59$, 60-69, 70-79 and 80+), stage (T1N0, T2N0, T3N0, T1N1, $\mathrm{T} 2 \mathrm{~N} 1$, T3N1), treatment (adjuvant chemotherapy, hormonal therapy or both combined (with or without radiotherapy), adjuvant radiotherapy and surgery only) and follow-up interval (<6 months, 6 months-4 years, 5-9 years and 10-14 years). Adjusted SIRs for metachronous CBC by age, stage, treatment and follow-up period were estimated with Poisson regression analysis. Poisson model fit was evaluated using the Pearson Chi-square goodness-of-fit test statistic. A high SIR does not necessarily imply a high disease burden, as incidence rates for specific age-groups may be low and the SIR is measured on a multiplicative scale. The absolute excess risk (AER), calculated by subtracting expected from observed CBCs and dividing this figure by the accumulated number of person-years (expressed per 10,000 person-years), better estimates the excess disease burden.

Metachronous CBC cumulative incidence and confidence intervals were estimated, with death or synchronous
CBC as competing risks [21, 22]. Cox Proportional Hazards $(\mathrm{CPH})$ analysis, accounting for competing risks, was used to examine the effect of initial treatment on the risk of developing a CBC [23]. A CPH model with a timedependent covariate, allocating follow-up time for each patient to the 'no CBC' group until CBC occurrence, was constructed to compare survival with and without $\mathrm{CBC}$. Variables considered in the model were age (continuous variable), morphology, stage, and treatment at index cancer diagnosis. Model fit was evaluated using residual-based graphical methods and goodness-of-fit test statistics. All reported $P$-values are two sided, the statistical significance level was set at a $P$-value $<0.05$.

\section{Results}

The cohort comprised 45,229 women with breast cancer. The median follow-up was 5.8 years (Inter Quartile Range 3.4-9.1 years) and 12,584 patients died during follow-up. The patients accumulated 295,097 personyears, 83,396 person-years for the 5-9 year period and 21,379 person-years for the period $\geq 10$ years after index cancer diagnosis. Only $0.1 \%$ of the patients were followed less than 6 months. In total 2,101 patients developed a $\mathrm{CBC}$, of which 624 were synchronous and 1,477 metachronous.

\section{Risk of synchronous CBC}

The frequency of synchronous CBC was $1.4 \%$ and increased with age $(P<0.001$, Table 1$)$. A very high SIR of synchronous CBC was observed for patients $<35$ years. The SIR was markedly lower for patients aged 35-70 years and increased again among those aged $\geq 70$. A logistic regression analysis showed an increase in risk of synchronous CBC increased with older age (Odds ratio (OR) for each 10-year increase 1.36; 95\% CI 1.28-1.45), presence of lymph node metastasis (OR 1.27; 95\% CI 1.07-1.49) and for lobular index cancer histology (OR $1.56 ; 95 \%$ CI 1.28-1.91).

Relative risk of metachronous CBC

The SIR was 2.5 (95\% CI 2.4-2.7) for all and 1.9 (95\% CI 1.8-2.1) for metachronous CBC. In univariate Poisson analysis the SIR of metachronous CBC decreased with age at index cancer diagnosis; the SIR was 11.4 (95\% CI 8.614.8) for women $<35$ years and 1.5 (95\% CI 1.4-1.7) for women aged $\geq 60$ years, respectively $\quad(P<0.001$, Table 1). 
Table 1 Number of patients (No), observed number (O), frequency (\%) and standardised incidence ratios (SIR) of synchronous CBC and observed number, 5- 10- and 15-year cumulative incidence (CR),
SIRs and absolute excess risk (AER) of metachronous CBC according to age, stage and treatment of the index cancer

\begin{tabular}{|c|c|c|c|c|c|c|c|c|c|c|c|c|}
\hline & \multirow[t]{2}{*}{ No } & \multicolumn{4}{|c|}{ Synchronous CBC } & \multicolumn{7}{|c|}{ Metachronous CBC } \\
\hline & & $\mathrm{O}$ & $\%$ & SIR & $95 \% \mathrm{CI}$ & $\mathrm{O}$ & SIR & $95 \% \mathrm{CI}$ & $\mathrm{AER}^{\mathrm{a}}$ & $\begin{array}{l}\text { 5-year } \\
\text { CR (\%) }\end{array}$ & $\begin{array}{l}\text { 10-year } \\
\text { CR }(\%)\end{array}$ & $\begin{array}{l}\text { 15-year } \\
\text { CR (\%) }\end{array}$ \\
\hline All patients & 45,229 & 624 & 1.4 & 10.4 & $9.6-11.3$ & 1,477 & 1.9 & $1.8-2.1$ & 26.8 & 2.1 & 4.2 & 5.9 \\
\hline \multicolumn{13}{|l|}{ Age } \\
\hline$<35$ & 1,198 & 13 & 1.1 & 81.7 & $43.5-139.8$ & 58 & 11.4 & $8.6-14.8$ & 72.8 & 2.9 & 6.1 & 7.4 \\
\hline $35-39$ & 1,999 & 7 & 0.4 & 9.7 & $3.8-20.9$ & 85 & 4.9 & $3.9-6.1$ & 53.8 & 2.9 & 4.6 & 7.0 \\
\hline $40-49$ & 9,121 & 78 & 0.9 & 9.7 & $7.6-12.1$ & 343 & 2.4 & $2.1-2.7$ & 34.4 & 2.4 & 4.5 & 6.9 \\
\hline $50-59$ & 11,179 & 129 & 1.2 & 8.6 & $7.1-10.2$ & 346 & 1.8 & $1.5-2.0$ & 22.5 & 1.9 & 4.3 & 6.1 \\
\hline $60-69$ & 10,386 & 140 & 1.4 & 8.5 & $7.1-10.0$ & 334 & 1.5 & $1.3-1.8$ & 18.3 & 1.8 & 4.2 & 6.1 \\
\hline $70-79$ & 8,056 & 159 & 2.0 & 11.6 & $9.8-13.6$ & 255 & 1.7 & $1.5-2.0$ & 25.3 & 2.1 & 4.0 & 5.3 \\
\hline $80+$ & 3,290 & 98 & 3.0 & 17.2 & $13.9-20.9$ & 56 & 1.2 & $0.9-1.6$ & 7.8 & 1.4 & 2.0 & 2.3 \\
\hline \multicolumn{13}{|l|}{ Stage $(T N M)$} \\
\hline T1 N0 & 18,474 & 225 & 1.2 & 9.0 & $7.8-10.3$ & 704 & 2.1 & $1.9-2.3$ & 32.0 & 2.4 & 4.9 & 7.2 \\
\hline T2 N0 & 8,862 & 130 & 1.5 & 10.6 & $8.8-12.7$ & 325 & 2.0 & $1.8-2.3$ & 31.5 & 2.3 & 4.4 & 6.2 \\
\hline T3 N0 & 439 & 5 & 1.1 & 8.4 & $2.7-19.6$ & 13 & 1.8 & $0.9-3.1$ & 24.4 & 2.1 & 2.8 & 4.3 \\
\hline T1 N1 & 7,160 & 99 & 1.4 & 10.9 & $8.8-13.4$ & 162 & 1.4 & $1.1-1.7$ & 10.9 & 1.4 & 3.2 & 4.8 \\
\hline $\mathrm{T} 2 \mathrm{~N} 1$ & 9,100 & 138 & 1.5 & 11.9 & $9.9-14.1$ & 220 & 1.6 & $1.4-1.9$ & 17.9 & 1.5 & 3.2 & 3.9 \\
\hline T3 N1 & 1,194 & 27 & 2.3 & 19.6 & $12.9-28.6$ & 53 & 3.7 & $2.8-4.9$ & 73.3 & 3.4 & 5.2 & 6.8 \\
\hline \multicolumn{13}{|l|}{ Adjuvant treatment ${ }^{\mathrm{b}}$} \\
\hline $\mathrm{CT}$ & 6,121 & 59 & 1.0 & 10.7 & $8.1-13.9$ & 177 & 2.3 & $1.9-2.7$ & 29.1 & 2.0 & 3.9 & 5.4 \\
\hline $\mathrm{HT}$ & 11,698 & 265 & 2.3 & 14.7 & $12.9-16.6$ & 233 & 1.1 & $0.9-1.3$ & 4.3 & 1.3 & 2.7 & 3.3 \\
\hline $\mathrm{CT}$ and $\mathrm{HT}$ & 2,564 & 38 & 1.5 & 14.7 & $10.3-20.2$ & 34 & 1.6 & $1.1-2.3$ & 14.3 & 1.2 & 4.5 & 6.5 \\
\hline RT (without CT/HT) & 13,044 & 91 & 0.7 & 5.3 & $4.2-6.6$ & 527 & 2.2 & $2.0-2.4$ & 34.4 & 2.4 & 4.9 & 7.5 \\
\hline No adjuvant treatment & 11,802 & 171 & 1.5 & 10.3 & $8.8-12.0$ & 506 & 2.2 & $2.0-2.5$ & 37.5 & 2.6 & 5.0 & 6.8 \\
\hline
\end{tabular}

a Absolute excess risk is expressed per 10,000 person years

${ }^{\mathrm{b}} \mathrm{CT}=$ chemotherapy, HT = hormonal therapy, RT = radiotherapy

Fig. 1 Standardised incidence ratios (SIRs) and 95\% confidence intervals for metachronous contralateral breast cancer, according to age at diagnosis and time since diagnosis of the index cancer

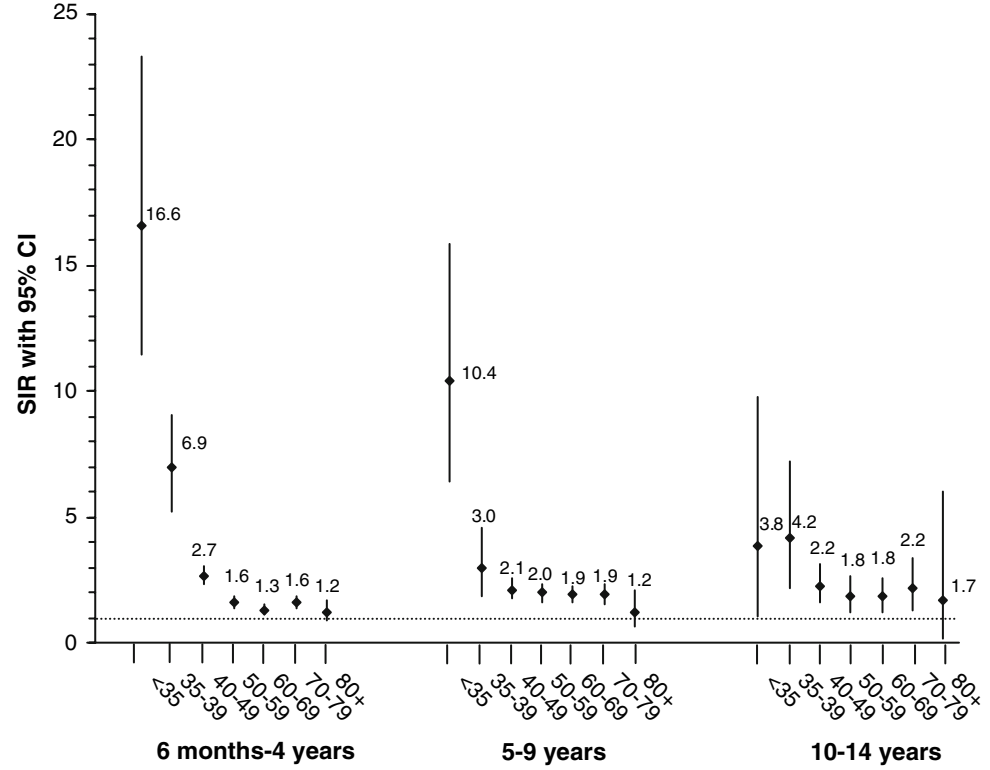


The SIR increased slightly with longer follow-up, from 1.8 after $0.5-4$ years to 2.0 after 5-9 years and 2.1 after 10-14 years of follow-up $(P=0.081)$. However, as Fig. 1 shows the age-specific SIRs for metachronous CBC actually decreased with follow-up time for patients younger than 40 years and increased only for older patients (interaction term age*follow-up period $P<0.001$ ).

Crude SIRs of metachronous CBC were 1.1 (95\% CI 0.9-1.3) following adjuvant hormonal therapy, 2.3 (95\% CI 1.9-2.7) following adjuvant chemotherapy and $2.2(95 \%$ CI 2.0-2.4) for patients not receiving hormonal or chemotherapy. Adjusted for age at index cancer diagnosis the SIRs for both hormonal (SIR 0.9; 95\% CI 0.6-1.2) and chemotherapy (SIR 1.1; 95\% CI 0.8-1.6) were in line with expected CBC numbers based on Dutch female population incidence rates, whereas the SIRs following surgery with/ without radiotherapy remained elevated (SIR 1.5; 95\% CI 1.1-2.1).

\section{Excess risk of metachronous $\mathrm{CBC}$}

The AER of developing a metachronous CBC was 26.8 per 10,000 woman-years (95\% CI 23.2-30.4). The AER decreased with age at index cancer diagnosis, from 72.7 (95\% CI 51.3-94.2) to 7.8 (95\% CI 7.7-23.4) per 10,000 person-years for women $<35$ years and $\geq 80$ years, respectively. Adjuvant hormonal therapy was associated with a very low AER of 4.3 (95\% CI -2.3-11.0) per 10,000 person-years.

Cumulative incidence of metachronous CBC

The cumulative incidence for metachronous CBC was $2.1 \%$ (95\% CI 1.9-2.3\%) at 5 years, $4.2 \%$ (95\% CI 3.9$4.5 \%$ ) at 10 years and $5.9 \%(95 \%$ CI 5.5-6.4\%) at 15 years of follow-up. The cumulative incidence increased at an average rate of $0.4 \%$ year. The highest cumulative incidence was observed for patients younger than 40 years (Fig. 2). The cumulative incidence increased with tumour size when positive lymph node metastases were present (Table 1). For patients receiving surgery only the 10-year cumulative metachronous $\mathrm{CBC}$ incidence was $5.0 \%(95 \%$ CI 4.5-5.5\%).

Hormonal therapy (HR $0.57, P<0.001$ ) and chemotherapy (HR $0.79, P=0.003$ ) were associated with a lower cumulative incidence of metachronous CBC compared to patients not receiving adjuvant systemic therapy in univariate $\mathrm{CPH}$, adjusted for competing risks. Combined adjuvant hormonal and chemotherapy was associated with a decreased risk of $\mathrm{CBC}(\mathrm{HR} 0.58, P=0.002)$, similar in magnitude to that for adjuvant hormonal therapy only.

In a multivariate $\mathrm{CPH}$, treatment with adjuvant hormonal (HR 0.58; 95\% CI 0.48-0.69) or chemotherapy (HR 0.73; 95\% CI 0.60-0.90), with or without radiotherapy, were associated with a reduced CBC risk, compared to no adjuvant systemic therapy (Table 2). The HR of a metachronous $\mathrm{CBC}$ increased with tumour size, but decreased with age at index cancer diagnosis. The histology of the index cancer was not associated with metachronous CBC risk. The HR of CBC did not differ between patients treated with surgery and radiotherapy or surgery only. However, in a multivariate $\mathrm{CPH}$ stratified for age, radiotherapy appeared associated with a, albeit non-significantly, increased CBC risk (HR 1.37 $P=0.111$, Table 2A) after adjusting for adjuvant hormonal and chemotherapy, age and stage. When the analysis was restricted to 5-year survivors, hormonal treatment was still associated with a decreased CBC risk (HR 0.65; 95\% CI 0.48-0.87, Table 2B).

Association of metachronous $\mathrm{CBC}$ with survival

Diagnosis of a CBC was associated with worse survival compared to patients without CBC. The HR of death
Fig. 2 Cumulative incidence of metachronous contralateral breast cancer by age at diagnosis of the index cancer

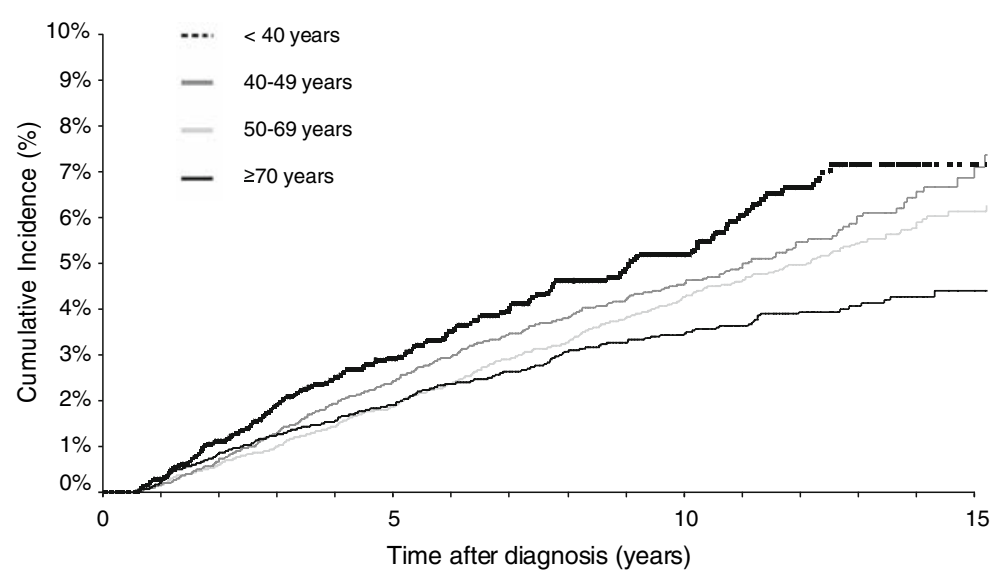




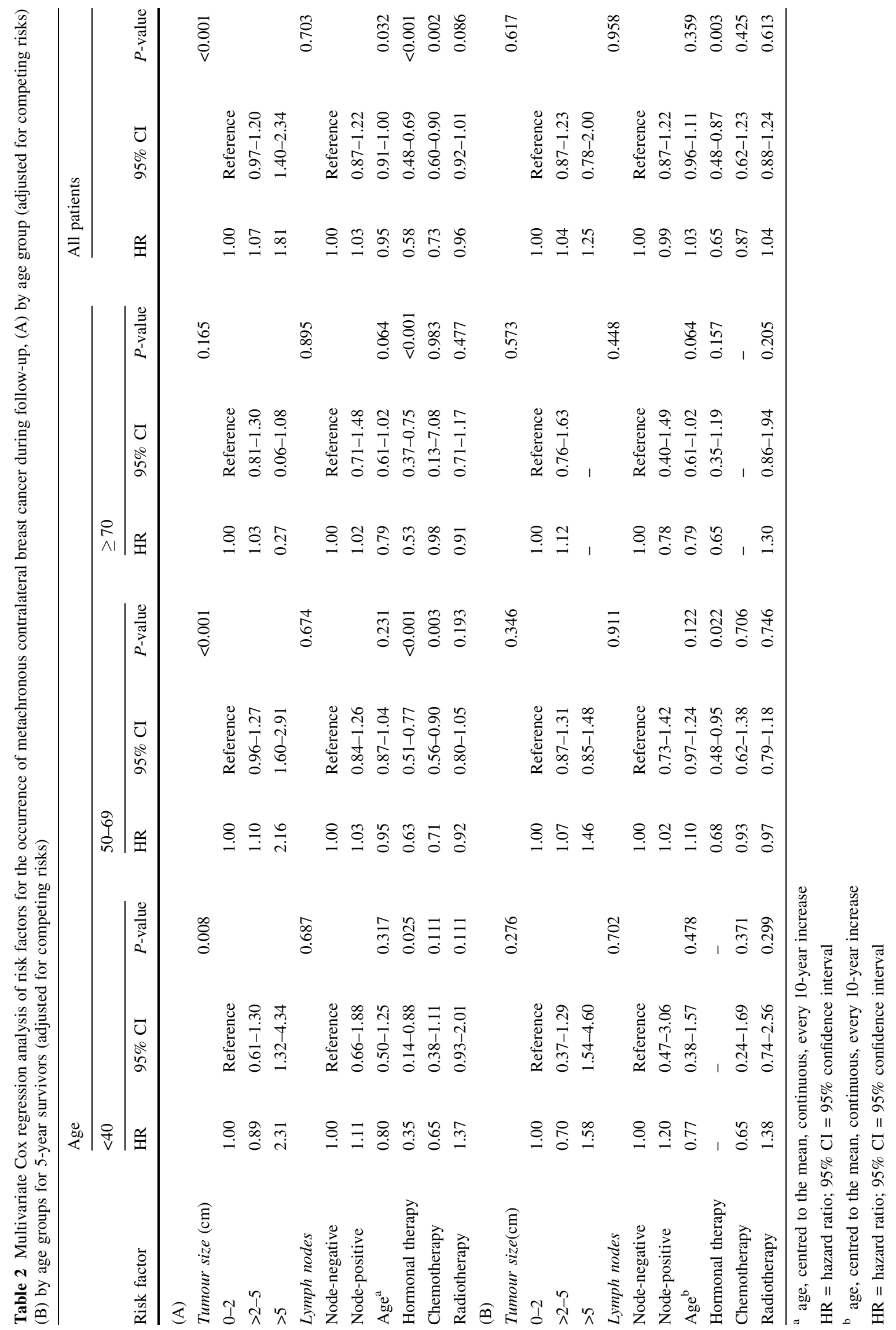


Table 3 Multivariate Cox regression analysis of overall survival, with $\mathrm{CBC}$ as a time-dependent covariate

\begin{tabular}{lccr}
\hline Risk factor & HR & $95 \%$ CI & $P$-value \\
\hline Stage of CBC (CBC yes versus no; time-dependent covariate) \\
Stage I & 0.99 & $0.86-1.15$ & 0.936 \\
Stage II & 1.32 & $1.13-1.54$ & $<0.001$ \\
Stage III/IV & 4.91 & $3.82-6.31$ & $<0.001$ \\
Stage unknown & 1.11 & $0.69-1.77$ & 0.661 \\
\hline
\end{tabular}

Adjusted for age and stage at index cancer diagnosis, both fitted as time-varying covariates

$\mathrm{HR}=$ hazard ratio $; 95 \% \mathrm{CI}=95 \%$ confidence interval

following CBC diagnosis was 1.44 (95\% CI 1.33-1.56) compared to patients without $\mathrm{CBC}$. Table 3 shows that the HR of death, adjusted for age and stage of the index cancer, differed according to CBC stage. While a stage I CBC did not increase the $\mathrm{HR}$ of death, stage II CBC increased the risk of death 1.3 fold.

\section{Discussion}

In this large population-based cohort comprising over 45,000 breast cancer patients we observed a 2.5-fold increased CBC risk. Assuming independence, the risk expressed in breast-years would be twice as high as in breast cancer survivors only one breast is at risk for CBC and the reference incidence rates concern cancer in any of two breasts. The cumulative incidence of metachronous CBC increased by $0.4 \%$ annually and did not level off even after 10 years of follow-up. Furthermore, a metachronous CBC in stage II or higher was associated with worse survival. Very high SIRs of CBC were observed for the youngest patients. Adjuvant hormonal and chemotherapy were associated with a $42 \%$ and $27 \%$ risk reduction of metachronous CBC, respectively. Adjusted for age, SIRs were not elevated in patients receiving adjuvant systemic therapy.

In our study $1.4 \%$ of the patients were diagnosed with synchronous CBC. Treatment does not likely affect the risk of synchronous $\mathrm{CBC}$, therefore this figure should be directly comparable between studies. The frequency reported in the literature varies between less than 1 and $3 \%$, largely depending on the time window chosen to define synchronous CBC [9, 24, 25]. Our definition of synchronous $\mathrm{CBC}$, diagnosis within 6 months of the index cancer, reflects the average time window in the literature. Differences in the diagnostic work-up, especially more frequent use of bilateral mammography, can also affect the frequency of synchronous CBC and may hamper comparison between studies [26, 27]. Lobular histology was also found associated with synchronous $\mathrm{CBC}$ occurrence, confirming earlier findings [8-10].
Several studies have reported a decrease in the risk of metachronous $\mathrm{CBC}$ with older age $[6,9,11]$. The very high SIRs of both synchronous and metachronous CBC for the youngest patients in our study were remarkable, with a more than 11-fold increased risk of metachronous and a more than 80-fold increased risk of synchronous CBC in patients aged $<35$. This was accompanied by the highest AERs, showing that these high SIRs cannot just be explained by a lower background incidence. Early onset breast cancer is a well recognized feature of hereditary breast cancer and mutations of the BRCA1 or BRCA2 genes strongly predisposes for CBC development [28-30]. Magnetic resonance imaging (MRI) may have a place in regular surveillance of these women [31]. Based on the results of our study all young women with breast cancer may be clear candidates for regular MRI screening directed at early $\mathrm{CBC}$ detection.

The 10-year cumulative incidence of metachronous CBC in our study (4.0\%) was relatively low compared to earlier studies [6-9]. This could be related to the increasing application of adjuvant systemic therapy over the last decades. In a study in patients diagnosed between 1975 and 1995 about $12.5 \%$ received hormonal therapy against about $32 \%$ in our study [9]. In our study hormonal therapy was associated with a reduction of metachronous CBC risk by $42 \%$. The reduction is comparable to the reduction seen in the EBCTCG meta-analysis and might be even higher than could be expected based on the EBCTCG meta-analysis, especially considering the fact that most of our patients received tamoxifen for 2 years only [13]. Our finding was also in line with the reduction of invasive breast cancer incidence observed in the NSABP tamoxifen prevention trial [32]. Aromatase inhibitors may even further reduce CBC risk [14-16]. Ongoing trials will determine the precise place of aromatase inhibitors, in terms of efficacy and safety, for the adjuvant treatment of breast cancer. CBC prevention will be an important endpoint in these studies. Interestingly, we also observed that adjuvant chemotherapy was associated with a $27 \%$ reduction of CBC. Although less than after hormonal therapy, this risk reduction was comparable to that seen in the polychemotherapy EBCTCG meta-analysis [17].

In our study relatively few patients received combined adjuvant systemic therapy and most were treated after 1997. Although our data do not allow firm conclusions about the effect of combined adjuvant systemic therapy, with a $42 \%$ proportional reduction of $\mathrm{CBC}$ occurrence combined systemic therapy appeared to be associated with a risk reduction similar to hormonal therapy alone.

Several studies have shown, that depending on the radiation technique used, the contralateral breast receives $1-13 \%$ of the applied dose to the treated breast, with the highest estimates reflecting scatter radiation to the medial 
part of the contralateral breast [33-35]. These doses are well within the tumourigenic range. Studies in atomic bomb survivors have shown decreasing radiation induced breast cancer risks with advancing age and no increased risk when irradiated over the age of 40 years [36].

Radiotherapy was not associated with a significantly increased CBC risk in our study. However, adjuvant systemic therapy is currently given to a high proportion of patients aged $<40$ years ( $56 \%$ in our study) and a protective effect of systemic therapy may well balance any increased risk caused by irradiation. The SEER data revealed an increased CBC risk associated with radiotherapy for patients surviving $\geq 5$ years, with a 1.3 fold risk increase in patients aged $<45$ years at index cancer diagnosis [6]. When restricted to CBCs diagnosed $\geq 5$ years after the index cancer, radiotherapy before the age of 40 years was not associated with $\mathrm{CBC}$ risk, although the risk estimate (HR 1.4) was comparable with SEER data. Possibly longer follow-up of our cohort may reveal an association between radiotherapy and $\mathrm{CBC}$ risk.

Our study showed that metachronous CBC stage II or higher was associated with worsened survival. Previously, patients who developed CBC were found to be at increased risk of disease recurrence and death $[6,8,24]$. An issue which may affect all studies of CBC risk is the difficulty in distinguishing $\mathrm{CBC}$ from metastases of the index cancer. However, several recent studies, which attempted to differentiate CBC between metastasis and second primary cancer using molecular-genetic techniques, did conclude that most localized metachronous CBC were distinct entities [37-39]. Most patients with metachronous $\mathrm{CBC}$ in our study were without evidence of distant metastases. However, metachronous CBC with distant metastases were not excluded and consequently CBC risk may have been slightly overestimated.

The strengths of our study derive from its populationbased character. The cohort includes a large number of patients with a nearly complete follow-up for vital status and, based on cancer registry data, the follow-up for CBC is virtually complete. Therefore this study provides a reliable estimate of the CBC risk and illustrates the potential of adjuvant treatment for CBC prevention.

Our study does harbour some limitations. Information on treatment for tumour recurrence was not recorded by the cancer registries. Furthermore, some underestimation of especially hormonal treatment may have occurred, as registration may sometimes have taken place before primary treatment was completed, and may have resulted in some underestimation of the effect of adjuvant treatment on CBC risk. However, studies using cancer registry data for evaluation of breast cancer treatment guideline adherence in the Netherlands showed generally good agreement between registry treatment data and suggested treatment by guidelines [40-42]. Longer follow-up is required to determine whether the prophylactic effect of tamoxifen on CBC occurrence will persist over time. Also, the effect of more prolonged tamoxifen (5 years instead of 2 years) and the effects of combined chemo- and hormonal therapy on CBC risk need further investigation.

As there is no evidence of a decreasing risk over time and metachronous $\mathrm{CBC}$ negatively affects patient's survival, regular long-term screening of the contralateral breast remains necessary. A combined approach with MRI and mammography appears most advisable for the youngest patient group. With broadening indications for adjuvant systemic treatment and new hormonal agents the $\mathrm{CBC}$ incidence may further decrease in the future.

\section{References}

1. Harvey EB, Brinton LA (1985) Second cancer following cancer of the breast in Connecticut, 1935-82. Natl Cancer Inst Monogr 68:99-112

2. Brenner H, Siegle S, Stegmaier C et al (1993) Second primary neoplasms following breast cancer in Saarland, Germany 19681987. Eur J Cancer 29a:1410-1414

3. Volk N, Pompe-Kirn V (1997) Second primary cancers in breast cancer patients in Slovenia. Cancer Causes Control 8:764-770

4. Evans HS, Lewis CM, Robinson D et al (2001) Incidence of multiple primary cancers in a cohort of women diagnosed with breast cancer in southeast England. Br J Cancer 84:435-440

5. Soerjomataram I, Louwman WJ, de Vries E et al (2005) Primary malignancy after primary female breast cancer in the South of the Netherlands, 1972-2001. Breast Cancer Res Treat 93:91-95

6. Gao X, Fisher SG, Emami B (2003) Risk of second primary cancer in the contralateral breast in women treated for early-stage breast cancer: a population-based study. Int J Radiat Oncol Biol Phys 56:1038-1045

7. Healey EA, Cook EF, Orav EJ et al (1993) Contralateral breast cancer: clinical characteristics and impact on prognosis. J Clin Oncol 11:1545-1552

8. Broet P, de la Rochefordiere A, Scholl SM et al (1995) Contralateral breast cancer: annual incidence and risk parameters. J Clin Oncol 13:1578-1583

9. Kollias J, Ellis IO, Elston CW, Blamey RW (2001) Prognostic significance of synchronous and metachronous bilateral breast cancer. World J Surg 25:1117-1124

10. Bernstein JL, Thompson WD, Risch N, Holford TR (1992) Risk factors predicting the incidence of second primary breast cancer among women diagnosed with a first primary breast cancer. Am J Epidemiol 136:925-936

11. Kollias J, Ellis IO, Elston CW, Blamey RW (1999) Clinical and histological predictors of contralateral breast cancer. Eur J Surg Oncol 25:584-589

12. Vaittinen P, Hemminki K (2000) Risk factors and age-incidence relationships for contralateral breast cancer. Int $\mathrm{J}$ Cancer 88: 998-1002

13. Early Breast Cancer Trialists' Collaborative Group (1998) Tamoxifen for early breast cancer: an overview of the randomised trials. Lancet 351:1451-1467

14. ATAC Trialists' Group (2002) Anastrozole alone or in combination with tamoxifen versus tamoxifen alone for adjuvant treatment of postmenopausal women with early breast cancer: first results of the ATAC randomised trial. Lancet 359: 2131-2139 
15. Baum M, Buzdar A, Cuzick J et al The ATAC (Arimidex, Tamoxifen Alone or in Combination) Trialists' Group (2003) Anastrozole alone or in combination with tamoxifen versus tamoxifen alone for adjuvant treatment of postmenopausal women with early-stage breast cancer: results of the ATAC (Arimidex, Tamoxifen Alone or in Combination) trial efficacy and safety update analyses. Cancer 98:1779-1781

16. Goss PE, Ingle JN, Martino S et al (2003) A randomized trial of letrozole in postmenopausal women after five years of tamoxifen therapy for early-stage breast cancer. N Engl J Med 349: 1793-1802

17. Early Breast Cancer Trialists' Collaborative Group (1998) Polychemotherapy for early breast cancer: an overview of the randomised trials. Lancet 352:930-945

18. Boice JD Jr, Harvey EB, Blettner M et al (1992) Cancer in the contralateral breast after radiotherapy for breast cancer. N Engl J Med 326:781-785

19. Hermanek P, Sobin LH (eds) (1992) International Union Against Cancer (UICC): TNM classification of malignant tumors. 4th edn, 2nd revision. Springer-Verlag; Berlin, Heidelberg, New York, London, Paris, Tokyo, Hongkong, Barcelona, Budapest

20. Sobin LH, Wittekind C (eds) (1997) International Union Against Cancer (UICC): TNM classification of malignant tumors 5th edn. Wiley-Liss, New York, Chichester, Weinheim, Brisbane, Singapore, Toronto

21. Gooley TA, Leisenring W, Crowley J, Storer BE (1999) Estimation of failure probabilities in the presence of competing risks: new representations of old estimators. Stat Med 18:695-706

22. Choudhury JB (2002) Non-parametric confidence interval estimation for competing risks analysis: application to contraceptive data. Stat Med 21:1129-1144

23. Lunn M, McNeil D (1995) Applying Cox regression to competing risks. Biometrics 51:524-532

24. Robinson E, Rennert G, Rennert HS, Neugut AI (1992) Survival of first and second primary breast cancer. Cancer 71:172-176

25. Heron DE, Komarnicky LT, Hyslop T et al (2000) Bilateral breast carcinoma: risk factors and outcomes for patients with synchronous and metachronous disease. Cancer 88:2739-2750

26. Mellink WA, Holland R, Hendriks JH et al (1991) The contribution of routine follow-up mammography to an early detection of asynchronous contralateral breast cancer. Cancer 67: 1844-1848

27. Roubidoux MA, Helvie MA, Lai NE, Paramagul C (1995) Bilateral breast cancer: early detection with mammography. Radiology 196:427-431

28. Dite GS, Jenkins MA, Southey MC et al (2003) Familial risks, early-onset breast cancer, and BRCA1 and BRCA2 germline mutations. J Natl Cancer Inst 95:448-457

29. de Sanjose S, Leone M, Berez V et al (2003) Prevalence of BRCA1 and BRCA2 germline mutations in young breast cancer patients: a population-based study. Int J Cancer 106:588-593
30. Loman N, Johannsson O, Kristoffersson U et al (2001) Family history of breast and ovarian cancers and BRCA1 and BRCA2 mutations in a population-based series of early-onset breast cancer. J Natl Cancer Inst 93:1215-1223

31. Kriege M, Brekelmans CT, Boetes C et al (2004) Magnetic Resonance Imaging Screening Study Group. Efficacy of MRI and mammography for breast-cancer screening in women with a familial or genetic predisposition. N Engl J Med 351:427-437

32. Fisher B, Costantino JP, Wickerham DL et al (1998) Tamoxifen for prevention of breast cancer: report of the National Surgical Adjuvant Breast and Bowel Project P-1 Study. J Natl Cancer Inst 90:1371-1388

33. Kelly CA, Wang XY, Chu JC, Hartsell WF (1996) Dose to contralateral breast: a comparison of four primary breast irradiation techniques. Int J Radiat Oncol Biol Phys 34:727-732

34. Warlick WB, O'Rear JH, Earley L et al (1997) Dose to the contralateral breast: a comparison of two techniques using the enhanced dynamic wedge versus a standard wedge. Med Dosim 22:185-191

35. Tercilla O, Krasin F, Lawn-Tsao L (1989) Comparison of contralateral breast doses from $1 / 2$ beam block and isocentric treatment techniques for patients treated with primary breast irradiation with 60CO. Int J Radiat Oncol Biol Phys 17:205-210

36. Tokunaga M, Land CE, Yamamoto T et al (1987) Incidence of female breast cancer among atomic bomb survivors, Hiroshima and Nagasaki, 1950-1980. Radiat Res 112:243-272

37. Janschek E, Kandioler-Eckersberger D, Ludwig C et al (2000) Contralateral breast cancer: molecular differentiation between metastasis and second primary cancer. Breast Cancer Res Treat $67: 1-8$

38. Imyanitov EN, Suspitsin EN, Grigoriev MY et al (2002) Concordance of allelic imbalance profiles in synchronous and metachronous bilateral breast carcinomas. Int J Cancer 100: $557-564$

39. Tse GM, Kung FY, Chan AB et al (2003) Clonal analysis of bilateral mammary carcinomas by clinical evaluation and partial allelotyping. Am J Clin Pathol 120:168-174

40. Voogd AC, van Beek MW, Crommelin MA et al (1994) Management of early breast cancer in southeast Netherlands since 1984. A population-based study. Regional Breast Cancer Study Group. Acta Oncol 33:753-757

41. Schaapveld M, de Vries EG, van der Graaf WT et al (2004) Quality of adjuvant CMF chemotherapy for node-positive primary breast cancer: a population-based study. J Cancer Res Clin Oncol 130:581-590

42. Ottevanger PB, Verhagen CA, Beex LV (1999) Quality of adjuvant chemotherapy in primary breast cancer in a non-trial setting. A comprehensive cancer centre study. The Breast Cancer Group of the Dutch Comprehensive Cancer Centre East (IKO). Eur J Cancer 35:386-391 